\title{
DYNAMIC TENSILE RESPONSE OF CAPRINE MUSCLES USING SPLIT HOPKINSON PRESSURE BAR
}

\author{
SOMNATH H. KADHANE ${ }^{* *} \&$ HEMANT N. WARHTAKAR ${ }^{2}$ \\ ${ }^{I}$ Research Scholar, Department of Mechanical Engineering, Dr. BabasahebAmbedkar Technological University, \\ Lonere-Raigad, Maharashtra, India \\ ${ }^{2}$ Associate Professor, Department of Mechanical Engineering, Dr. Babasaheb AmbedkarTechnological University, \\ Lonere-Raigad, Maharashtra, India
}

\begin{abstract}
Computer modeling and numerical simulation has become an efficient diagnostic tool to predict the human body injuries caused due to high speed automotive impacts, blast and ballistic impacts. Soft tissues such as muscles and skin in human body are exposed to varying strain rates under dynamic loadings during impacts. The prediction of impact-induced injuries requires a thorough understanding of mechanical behaviour of soft tissues for computational modeling of human body. In the present study, uniaxial tensile tests were conducted on caprine lower extremity muscles in thestrain rate range of $\left(500 \mathrm{~s}^{-1}-3500 \mathrm{~s}^{-1}\right)$ using custom-made split Hopkinson pressure bar (SHPB) apparatus. The difficulties in the dynamic testing of delicate tissues, for example, estimation of frail transmitted signs, utilization of viscoelastic weight bars, tractable stacking of example and age of consistent strain rate were tended to in powerful elastic testing of delicate tissues utilizing polymeric SHPB. Polymeric bars were utilized in SHPB as impedance is nearer to the delicate tissues to create quality transmitted signs during experimentation. The lessening and scattering in waves in polymeric bar during sway are remedied utilizing segregated occurrence bar tests. The pressure strain results were resolved from the reproduced waves for the tests directed on lower furthest point caprine muscles. The muscle examples were tried along and opposite to the fiber course to examine the directional reliance of tissue conduct. The pressure strain reaction was seen as non-straight and huge dependant on strain rate when tried along and opposite to fiber course at same strain rates. It is additionally seen that at a similar strain rate, the example worry of caprine muscle along the opposite fiber heading is higher than that along the fiber course.The obtained results may further be used to develop finite element human body models and safety systems for human body in high rate scenario.
\end{abstract}

KEYWORDS: Tensile Loading, SHPB, Strain Rate, Impact \& Tissue Behaviour

Received: May 11, 2020; Accepted: Jun 01, 2020; Published: Jul 16, 2020; Paper Id.: IJMPERDJUN2020347

\section{INTRODUCTION}

Higher strain rates occur not only in automobile impacts, but also in airplane accidents, impact or bird-strike events, sport accidents, industrial accidents, slip and falls, explosive blast, ballistic and bullet impacts. A high strain rate response of muscle tissue is essential for computer modeling of soft tissue in research and application activities of impact biomechanics. The mechanical response of soft biological tissues at different strain rates is also essential in high speed automotive impacts and crash analysis to develop human body models and to design body armors for effective understanding of the injury mechanisms. Lower extremity muscles of human body are subjected to compressive impact during pedestrian-vehicle impacts. The muscle constitutes are compressed in the direction transverse to the fiber orientation and slide over each other, while the muscle constitutes are stretched in the 
direction parallel to the fiber orientation. Since muscles are formed of fiber bundles, the tensile behavior will be different than the compressive behavior.

The available quantitative data on the mechanical response of biological soft tissues is mostly limited to quasistatic strain rates $\left(\dot{\varepsilon}<0.1 \mathrm{~s}^{-1}\right)$ [1-4]. The response of soft tissues at intermediate strain rates $\left(\dot{\varepsilon}>5 \mathrm{~s}^{-1}\right)$ have also been studied for bovine medial collateral ligament [2], human muscle [4], human spleen [5], human diaphragm [6] and human aortic tissue [7] under tensile loading. The response of soft tissues at intermediate strain rates $\left(\dot{\varepsilon}>5 \mathrm{~s}^{-1}\right)$ have been studied for skeletal muscle [1], porcine muscle [3], bovine liver tissue [14] and adipose tissue [17] under compressive loading. Studies on muscle tissue responses under dynamic loadings at higher strain rates are limited in available literature. SHPB, originally developed by Hopkinson [89] has been critically studied by Davis [10] and later improved by Kolsky [11] with two pressure bars connected in series. SHPB has been widely used to characterize the dynamic behavior of ductile materials in the strain rate range of $10^{2}-10^{4} \mathrm{~s}^{-1}$ [12]. This strategy has been altered for testing delicate tissues under compressive, pliable and torsional stacking. A couple of examination endeavors have been put resources into the dynamic portrayal of delicate tissues at higher strain rates because of trial issues in the portrayal of delicate tissues. Longer than 10 years, SHPB with low impedance metallic bars has been broadly used to research the dynamic pressure strain reaction of delicate tissues under compressive stacking and such reaction has been found to totally different structure the semi static reaction. The compressive pressure strain reactions of cow-like delicate tissues, for example, kidney tissue [13], liver tissue [14], mind tissue [15]; porcine delicate tissues, for example, liver tissue [16], fat tissue [17], trachea tissue [18] and lung tissue [19] have been as of late concentrated over wide scope of strain rates utilizing adjusted renditions of low impedance metallic SHPB. The compressive mechanical reaction of porcine muscle [20] and ox-like muscle [21] utilizing low impedance metallic bars and PMHS muscle [22] and goat muscle [23] utilizing polymeric SHPB has been concentrated methodicallly at high strain rates. The test results uncovers that the delicate tissue reactions were non-straight and dependant on strain rate.

Experiments to obtain the mechanical response of soft tissues at high strain rates under tensile loading have been limited and less tensile data is reported in literature as it is very challenging to subject the soft tissue specimen to valid dynamic loading conditions and measure their mechanical responses. The ductile pressure strain reactions of porcine muscle [24-25], cow-like ligament [26], pig skin [27-28] and human cervical spine tendons [29] have been as of late concentrated over a wide scope of strain rates utilizing changed renditions of SHPB. Tests of delicate tissue reactions under strain are scant [24-29]. An exact and reasonable material model that speaks to the dynamic reaction of delicate tissues requires more information on tractable reaction of delicate tissues at high strain rates. The shear reaction of ox-like cerebrum tissue [30] and porcine lung tissue [19] has additionally been concentrated under torsional stacking utilizing changed SHPB with shear hold connection. The porcine muscles were tested at a strain rate range of 700-2100 s-1 [24], the bovine tendons were tested at a strain rate of $2500 \mathrm{~s}^{-1}[26]$ and the pig skin tissues were tested in the strain rate range of $1700-3500 \mathrm{~s}^{-1}[28]$.

The major challenges in characterization of soft tissues are low signal to noise ratio, use of low impedance pressure bars, the attainment of dynamic stress equilibrium and constant strain rate in specimen and generation of low amplitude repeated incident pulse. The remedial solutions to these challenges as suggested in literature [31] has been incorporated in custom-made polymeric SHPB for tensile testing of soft tissue. The weak signals are propagated through transmission bar during soft tissue testing using metallic SHPB due to acoustical impedance and geometrical mismatch of 
pressure bars and specimen. This can be improved by using low-impedance metallic bars such as titanium, aluminum and magnesium alloy bars and hollow transmission bar. The utilization of empty transmission bar presents vulnerability in readings as signs engendering through strong bar and empty bar are not same. The polymeric SHPB empowers the estimation of low-stress flags and permits the review of pressure balance in the test example. The example is exposed to longer test time because of the lower sound speeds when contrasted with the low-impedance metallic bars, and in this manner empowers enormous strains applied before the impacts of reflected pressure waves. This gives the uniform disfigurement of the example. The advantages of polymeric SHPB are excellent signs might be estimated on the weight bars because of impedance coordinating between the weight bars and delicate tissues so that and adaptability in example geometry because of longer pressure wave rise time for the PMMA material. The versatile investigation of SHPB can't be legitimately applied for polymeric bars as polymeric bars are viscoelastic in nature. The pressure wave weakens and scatters in the medium because of the utilization of polymeric bars in SHPB. The strain estimated at the center area of the weight bar is not the same as the strain estimated at the interface of bar-example.

In the present study, the challenges in the dynamic testing of soft tissues such as measurement of weak transmitted signals, use of viscoelastic pressure bars, tensile loading of specimen and generation of constant strain rate were addressed in dynamic tensile testing of soft tissues using polymeric SHPB. We used polymeric pressure bars to obtain dynamic stress-strain response of caprine muscle tissues and wave correction was applied. The Labview assisted data acquisition system was used to acquire the stress waves propagating through the pressure bars during impact. Matlab program based on viscoelastic analysis of SHPB was used for dynamic characterization of muscle tissues. Polymeric SHPB setup and Matlab program has been validated. The experimental protocol in dynamic testing of soft tissues has been adopted from previous studies [22-24]; however the protocol to prepare the caprine muscles specimens for tensile testing was developed in-house. The muscle specimens were tested along and perpendicular to the fiber direction to study the directional dependency of tissue behaviour. The stress-strain response of caprine muscle tissues along and perpendicular to muscle fiber direction is obtained by using custom-made polymeric SHPB under tensile loading at varying strain rates. These experimental tests at higher loading rates of 400-3700 s-1 are necessary to understand the tissue behavior and injury patterns not only during automobile accidents but also during blast or ballistic impacts. During military operations, war-fighters are subjected to blast events. In response to this, a bulletproof jacket is an important protection for the human body from high velocity, short duration blunt and ballistic impacts.

\section{MATERIALS AND METHODS}

\section{Specimen Preparation}

The preparation of specimen and gripping method to avoid the failure of soft tissue at the grips is also a challenging task. This motivates the study on muscle tissue characterization using modified SHPB with polymeric pressure bars. The new muscle examples from lower furthest point of three Indian grown-up goats were gathered from a butcher house. All the examples were put away in saline arrangement at room temperature about $20^{\circ} \mathrm{C}$ for three hours before testing. All the examples were defrosted at room temperature for under three hours before testing. The sash layer was expelled from the muscles. The measures were taken to forestall the lack of hydration of the muscle tissue during experimentation. Examples were not preconditioned preceding testing. The physical stacking of muscle tissue was stayed away from till playing out the tests. 


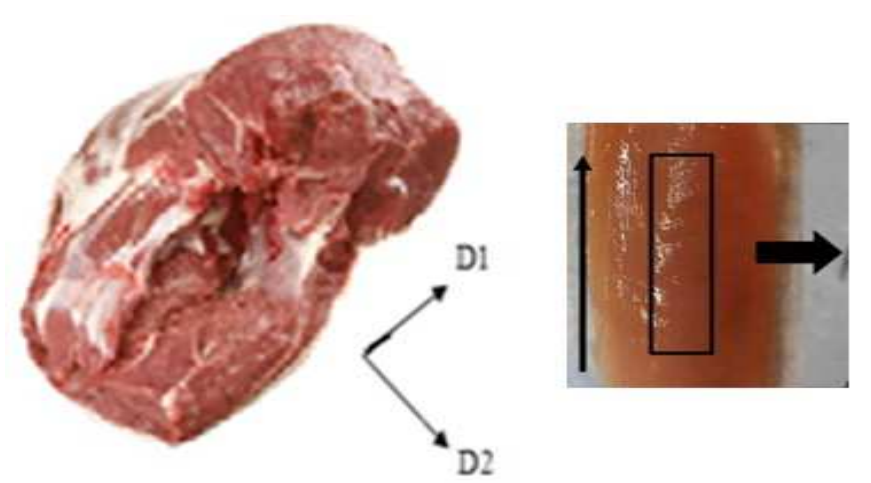

Figure 1: Thin Muscle Sheet Preparation.

The preparation of muscle sheet for tensile specimen is represented in figure 1. The level muscle sheet is utilized to set up a dainty rounded example for decreasing the outspread latency impacts delivered during hub speeding up of example $[30,35]$. The muscles were cut into $3 \mathrm{~mm}$ thick level sheets along and opposite to the fiber direction of muscle tissue. The width (level bolt) and length (vertical bolt) of the level sheet is kept up as $30 \mathrm{~mm}$ and $50.3 \mathrm{~mm}$. The width of the solid sheet is set up along $\left(D_{1}\right)$ and opposite $\left(D_{2}\right)$ to the muscle fiber course. The muscle level sheets must be then marginally broadened and smoothed when they are folded over the test segment and clipped at the two closures of the polymeric compel bars so as to maintain a strategic distance from the filaments of muscle to wrinkle. So as to stack the muscle along and opposite to the fiber heading, the barrel shaped hub of the cylindrical muscle example is kept along the fiber bearing and opposite to the muscle fiber course.The cylindrical axis of specimen is the direction of tensile loading during the tensile tests.

\section{Specimen Gripping}

The flat muscle sheets are then wrapped around the test section with predetermined gap between incident and transmission bars. The predetermined gap of $2 \mathrm{~mm}$ obtained after trial experiments is used to clamp the muscle sheet around the pressure bar to facilitate axial stress equilibrium. Flat muscle sheet is used as tubular specimen to reduce radial inertia effects. The internal surface of tubular specimen was bonded to cylindrical surface of pressure bar, whereas the outer surface of tubular specimen was clamped at both ends using plastic snap clamps. An illustration of the tubular muscle specimen mounted in the test section is shown in figure 2.

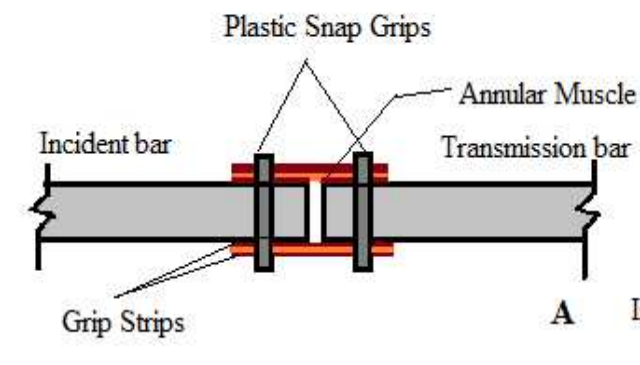

(A) 2-D view

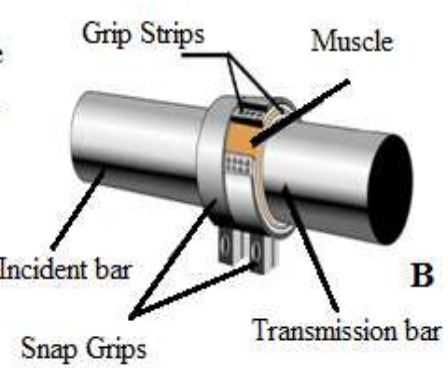

(B) 3-D view

Figure 2: Thin Annular Muscle Specimen Mounted between Pressure Bars During Tensile Experiments.

The reflected pulse in the incident bar might be affected due to the use of the clamps for mounting of the specimen in between pressure bars under tensile loading. However, the reflected pulse is affected very less and its effect is 
negligible due the use of plastic hose clamps [31]. The flat sheet was slightly stretched to avoid the wrinkling of muscle fibers while attaching the specimen on pressure bar ends. However, the effect of stretching of specimen, use of plastic snap grips and the discontinuity at junction point where the flat muscle sheet meets itself to form tubular geometry of specimen are not studied. This is a limitation of the present study.

\section{Experimental Setup}

The schematic of tensile SHPB is shown in figure 3 consists of incident bar, transmission bar and hollow striker bar. All the pressure bars were made from PMMA (Poly-methyl Metha-acrylate). Theincident and transmitter pressure bars has diameter $16 \mathrm{~mm}$ and length $1220 \mathrm{~mm}$. The empty striker bar with inner distance across of $16 \mathrm{~mm}$ and outer measurement of $32 \mathrm{~mm}$ has been utilized as shot to produce for pliable stacking of the example during exploratory tests. The rounded example is cinched with foreordained hole among occurrence and transmitter bar by utilizing plastic snap clips and the episode stress beat is produced by the effect of empty striker bar on the neckline joined to the occurrence bar. The occurrence and reflected signs were estimated by a couple of resistive foil strain measures (Omega Engineering) mounted the episode bar and transmission bar at in the center. The speed of striker bar shot is constrained by pressurized packed air that is constrained into the launcher chamber. PTFE (Polytetrafluoroethylene) meager plates as heartbeat shapers were utilized to get the dynamic pressure harmony inside the example. At the point when terminated utilizing snappy acting solenoid valve, the launcher discharges the air and moves a striker bar into the finish of occurrence bar. The impact of striker bar with the neckline toward the finish of episode bar makes the occurrence beat (malleable) that engenders through the occurrence bar towards the example. The episode beat is recorded by the strain measures mounted on the occurrence bar. When the wave scopes to the example, it parts into two heartbeats viz. transmitted wave and reflected wave. The transmitted heartbeat (elastic) goes through the example and into the transmitter bar where the vitality is recorded by the strain checks mounted on transmitter bar. The subsequent heartbeat (compressive) is reflected away from the example and goes down to the episode bar. Toward the finish of SHPB, a stop bar retains the effect of transmission bar to finish the test.

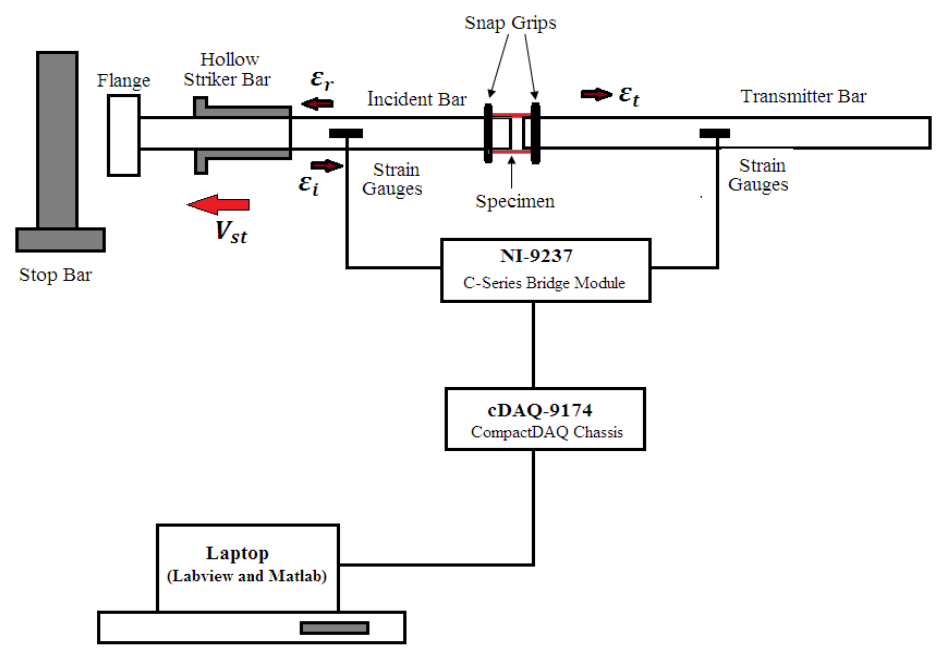

Figure 3:Schematic of Tensile Polymeric SHPB Setup.

One dimensional wave propagation theory in elastic pressure bars along axial direction yields the calculation of strain $\left(\varepsilon_{s}\right)$ strain rate $\left(\dot{\varepsilon}_{s}\right)$ and stress $\left(\sigma_{s}\right)$ induced in the specimen using following equations. 


$$
\begin{aligned}
& \varepsilon_{s}=\frac{-2 C_{0}}{L_{S}} \int_{0}^{t} \varepsilon_{r} d t \\
& \dot{\varepsilon_{S}}=\frac{-2 C_{0}}{L_{S}} \varepsilon_{r} \\
& \sigma_{S}=\frac{A_{0}}{A_{S}} E \varepsilon_{t}
\end{aligned}
$$

Where $\varepsilon_{r}$ and $\varepsilon_{t}$ are the reflected and transmittedstrain histories, respectively; $A_{0}$ is the cross-sectional area or pressure bars; $C_{0}$ and $\mathrm{E}$ are the sound wave speed and modulus of elasticity in the pressure bar, respectively; $A_{S}$ and $L_{S}$ are the initial cross-sectional area and length of specimen, respectively.

An experimental method suggested by Bacon [32] has been applied to account the strain mismatch for the correction of wave attenuation and dispersion. The propagation coefficients obtained from free end tests on the incident bar at $2 \mathrm{~m} / \mathrm{s}$ using the viscoelastic analysis of polymeric SHPB has been used in muscle tissue characterization. The constriction coefficient and wave number were resolved utilizing adequacy and stage range of occurrence and reflected heartbeats individually. The wave numbers at various frequencies were utilized to discover the stage speeds. The reflected heartbeat and transmitted heartbeat is recreated by fusing the weakening and scattering revision in the particular estimated beats for the separation it has engendered. The count of example stress, strain and strain rate in the recurrence space was finished utilizing viscoelastic investigation for polymeric bars, where the stage speed is an element of recurrence.Stress $\tilde{\sigma}_{S}(\omega)$, strain, $\varepsilon_{S}(\omega)$ and strain rate, $\dot{\varepsilon}_{S}(\omega)$ in the specimen are calculated using reconstructed waves in frequency domain.

$$
\begin{aligned}
& \dot{\varepsilon}_{S}(\omega)=-\frac{2 \tilde{\varepsilon}_{R} \_n e w}{L_{0}}[\omega)\left[\frac{i \omega}{\gamma(\omega)}\right] \\
& \varepsilon_{S}(\omega)=\int_{0}^{t} \dot{\varepsilon}_{S}(\omega) d t \\
& \tilde{\sigma}_{S}(\omega)=\frac{A_{0} \tilde{\varepsilon}_{T} n e w(\omega)}{A_{S}} E^{*}(\omega)
\end{aligned}
$$

Using inverse Fourier transform, stress $\sigma_{S}(\omega)$, strain $\varepsilon_{S}(t)$ and strain rate $\dot{\varepsilon}_{S}(t)$ the specimen are then calculated in time domain.

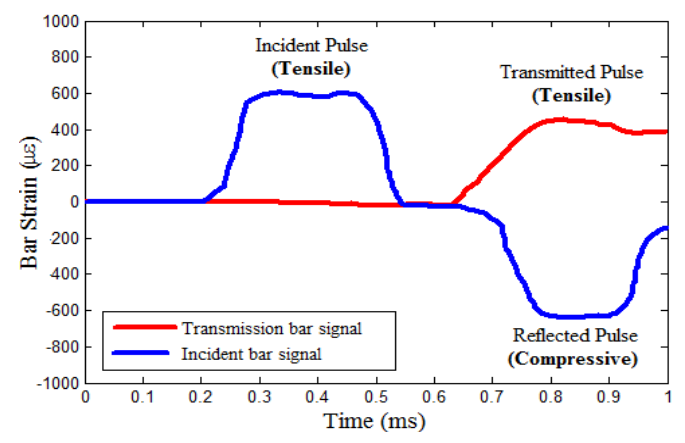

Figure 4: Wave form History During Tensile Experiments on

Tubular Caprine Muscle Specimen using Polymeric SHPB. 
The incident, reflected and transmitted stress pulses during tensile experiments on caprine muscle specimen at higher strain rates are shown in figure 4. The frequency oscillations and initial spikes observed in measured stress pulses during experiments using elastic SHPB are nearly eliminated in viscoelastic SHPB tests due to use of pulse shaping and polymeric pressure bars. This facilitates accurate characterization of stress-strain response at higher strain rates under dynamic loading.The occurrence and reflected heartbeat are about same and inverse in sign due the mechanical impendence of the delicate tissue is nearer to the weight bars. The transmitted heartbeat records the pressure history and reflected heartbeat records the strain history in the example. The reflected heartbeats recorded by occurrence strain checks and the segment of compressive heartbeats that proceeds through the example recorded by transmission strain measures were utilized to ascertain the strain and stress initiated in the muscle example.

\section{Experimental Protocol}

A total 40 tubular muscle specimens (20 along the fiber direction and 20 along the perpendicular fiber direction) prepared from lower extremity muscles of the caprine were testes under dynamic tensile loading at four strain rates of $500 \mathrm{~s}^{-1}, 1000$ $\mathrm{s}^{-1}, 1700 \mathrm{~s}^{-1}, 2700 \mathrm{~s}^{-1}$ and $3500 \mathrm{~s}^{-1}$ in each direction along and perpendicular to the muscle fiber orientation. At each strain rate,the tests were repeated at least 4 times under identical loading conditions to study the repeatability of experimental results.The just one test was directed on each example. For each test, the cylindrical muscle example was braced between the occurrence bar and transmitter bar utilizing plastic snap clasps. All the tests were performed till disappointment of example in unconfined condition. The unconfined condition implies the examples were permitted to amplify openly the longitudinal way till disappointment and agreement the sidelong way. All the test tests were led at room temperature (20$\left.25^{\circ} \mathrm{C}\right)$.

\section{Data Analysis}

LabVIEW assisted data acquisition system composed of NI 9237 bridge module and NIcDAQ 9174 with LabVIEW 2014 software has been established to acquire and records the stress wave history using strain gauges mounted on the pressure bars. The incident and transmission bar signals were captured using incident and transmission strain gauges at $50 \mathrm{kHz}$ for 4 milliseconds resulting in 200 data points per test. During processing, the signal conditioning and amplification was done using continuous sampling acquisition mode to make signal suitable for processing. The waveform data was filtered at 10 $\mathrm{kHz}$ during data acquisition using low pass filter for the reduction of noise. The signal to noise ratio (SN ratio) was evaluated for the acquired waveforms and tests with SN ratio lower than 10 were excluded for further analysis [22]. The MATLAB program based on viscoelastic analysis was used to post process the recorded waveform. This enables the calculation of the propagation coefficient, reconstruction of the waveform at specimen-bar interface and determination of the stresses, strains and strain rates in the muscle specimen to plot the stress-strain response at each strain rate. 


\section{RESULTS AND DISCUSSIONS}

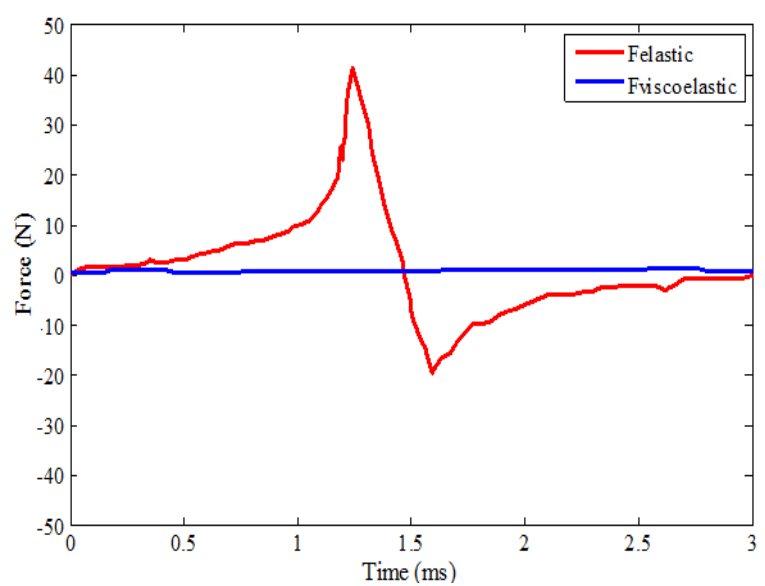

Figure 5: Forces Determined at Free end of Bar using Elastic and Viscoelastic Analysis of SHPB.

An experimental approach as suggested by Bacon [32] has been implemented for the viscoelastic analysis of polymeric SHPB and it was found that phase velocity increases with increase in frequency. The forces at the free end form the measured strains has been calculated using elastic and viscoelastic analysis of SHPB as suggested by to validate the test methodology [32]. By using elastic analysis, forces were calculated at the free end $\left(\mathrm{F}_{\text {elastic }}\right)$ from the strains measured at strain gauge mounted location. In this method, the phase velocity c $(\omega)$ was considered to be independent of the frequency and the attenuation coefficient $\alpha(\omega)$ was assumed to be zero at all frequencies implying that there was no attenuation and dispersion in the waves across its travel, in other words the bar material was considered to be pure elastic. To implement this condition, phase velocity $\mathrm{c}(\omega)$ was kept constant at all frequencies while calculating the wave number $\mathrm{k}(\omega)$. In the second method, viscoelastic analysis was performed to calculate the forces $\left(\mathrm{F}_{\mathrm{visco}}\right)$ at the free end by considering the attenuation and dispersion in waves from the measured strain gauge location. Therefore, the forces at the free end were calculated from the strains measured at gauge location by subjecting the propagation distance as $\mathrm{d}=610 \mathrm{~mm}$. Hence, this analysis considers the frequency dependency of the phase velocity and attenuation coefficient. The forces obtained are then compared in the figure 5 and it was found that the forces at the free end determined using viscoelastic analysis is nearly zero. This validates the polymeric SHPB and can be implemented for soft tissue characterization. It has also been observed that the maximum force of $40 \mathrm{~N}$ developed at free end due to elastic analysis and zero force is developed at free end due to viscoelastic analysis. An elastic analysis will give erroneous results if elastic analysis is used for the determination of stress-strain response for the SHPB with viscoelastic pressure bars. Hence, elastic cannot be directly used for viscoelastic SHPB with polymeric bars.

The typical time histories of strain and strain rate of muscle tissue at strain rate of $3500 \mathrm{~s}^{-1}$ is shown in figure 6 . The nearly flat top portion indicates that the muscle specimen deformed at a nearly constant strain rate. The resultant average tensile stress-strain curves are depicted in figures 7 and figure 8 for the loading directions along and perpendicular to the muscle fiber direction respectively. Each muscle specimen was tested at five impact velocities corresponding to five strain rates form 500-3500 s-1 up to failure. Each test was repeated at least four times to obtain stress-strain curves at similar loading conditions and each muscle specimen was tested once to the point of failure to investigate the effects of rate dependence. It is noted that each of the curve in the two figures represent the average of at least four repeated experimental tests performed under same loading conditions. The response of caprine muscle tissue was non-linear in tensile loading for 
all loading rates.

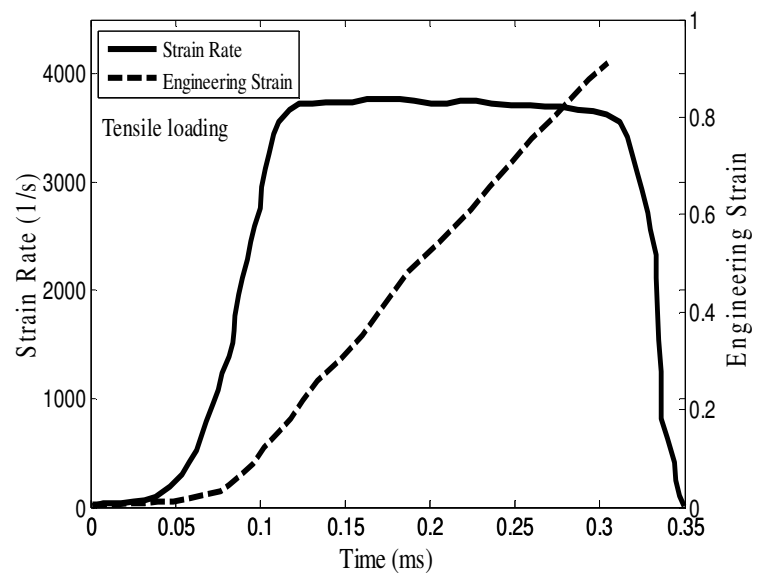

Figure 6: Strain and Strain Histories in the Caprine Muscle Specimen.

The aftereffects of the current investigation additionally indicated that the reaction of muscle tissue differed concerning higher unique stacking rates, with higher rate tests giving higher disappointment stresses and higher disappointment strains. The expansion in applied strain rate prompts the expansion in the work solidifying impact of stream pressure. There is nonattendance of toe area toward the starting which demonstrates that the muscle filaments are straight and smooth toward the beginning of the stacking. The expansion in applied strain rate prompts the increment in the work solidifying impact of stream stresses. The pressure strain bends shows that the muscle tissue reaction is touchy to both strain and strain rate.

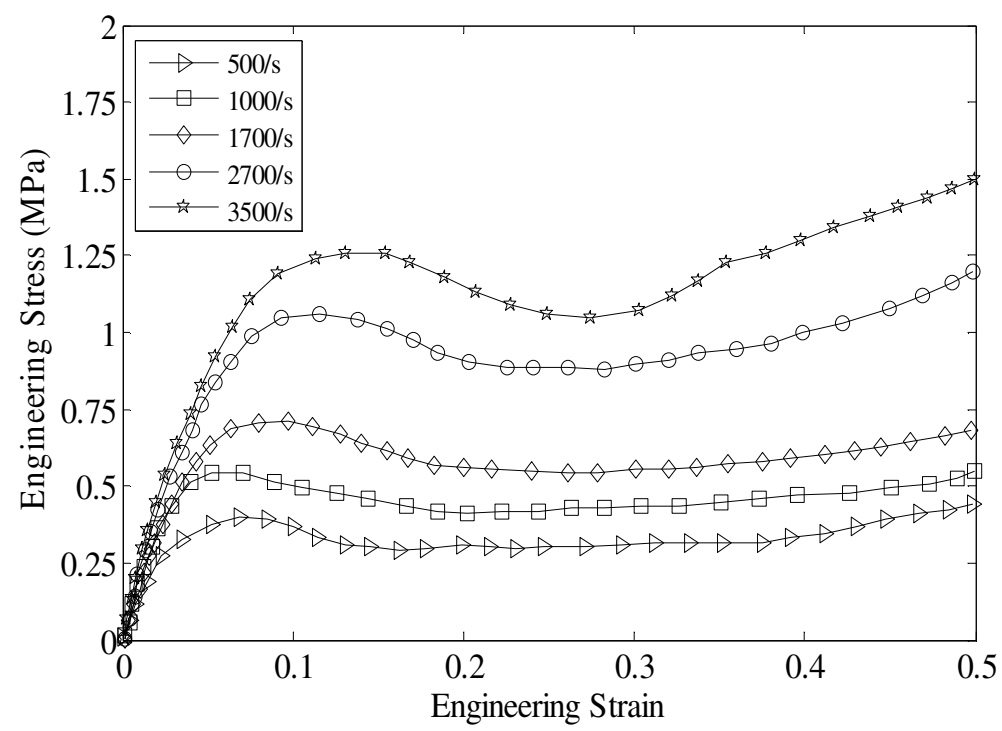

Figure 7: Average Stress-Strain Curves of the Caprine Muscle along the Fiber Direction at Various Strain Rates. 


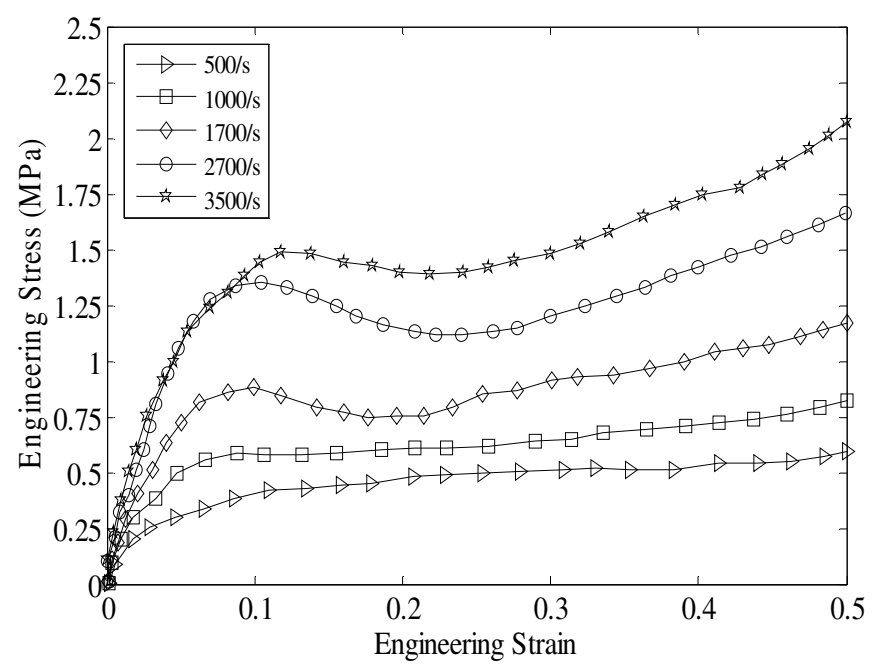

Figure 8: Average Stress-Strain Curves of the Caprine Muscle Perpendicular to the Fiber Direction.

For both fiber direction cases, the steep initial rise is considered to be first phase in the stress-strain response. In the second phase, strain starts to accumulate rapidly without profound increase in stress upto failure.The similar trends of tensile stress-strain curves were reported in previous studies on porcine muscle at quasi-static and high strain rates [30] and human muscles at intermediate strain rates [10]. Authors were not studied the quasi-static response of the caprine muscle. However, experimental data from the previously published study [24] at quasi-static strain rate of $0.5 \mathrm{~s}^{-1}$ were compared with the stress-strain data at dynamic strain rates in the present study as shown in figure 6 and figure 7 . The specimens used in current study were approximately similar to those as used in Nie et al. study. It was found that the stress-strain response at quasi-static loading showed a similar nature when compared with current SHPB response at dynamic loading.

The vast majority of the past investigations show that the muscle conduct is viscoelastic [1, 35-36] and viscoelasticity shown during ductile stacking is likely because of the inactive properties of protein titin [37-38]. Be that as it may, not many examinations watched the muscle conduct at different strain rates. The consequences of the current examination show that the pressure strain reaction is unequivocally subject to strain rates applied. It is additionally seen that at a similar strain rate, the example stress opposite to fiber course (TR) was higher than that along the fiber heading (AL). This might be because of various power creation instruments for the muscle along and opposite to the fiber heading. Muscle strands are produced using myofibrils. Myofibrils are made out of sarcomeres masterminded in arrangement and every sarcomeres has covering myosin and actin fibers. The fiber-formed fascicles and perimysium turns into the heap bearing component if the muscle is stacked along the fiber heading and opposite to the fiber course separately. The stretchopposing trait of perimysium may bring about the higher distortion in the opposite muscle fiber heading [32].

Table 1:Stress Values at Various Strain Rates Observed in Caprine Muscle along and Perpendicular to Muscle Fiber Direction at $40 \%$ Strain

\begin{tabular}{|c|c|c|}
\hline \multirow{2}{*}{ Strain Rate $\left(\mathbf{s}^{-\mathbf{1}}\right)$} & \multicolumn{2}{|c|}{ Stress (MPa) at 40\% Strain } \\
\cline { 2 - 3 } & $\mathbf{A L}$ & $\mathbf{T R}$ \\
\hline 500 & 0.3 & 0.5 \\
\hline 1000 & 0.45 & 0.7 \\
\hline 1700 & 0.6 & 1 \\
\hline 2700 & 1 & 1.4 \\
\hline 3500 & 1.3 & 1.75 \\
\hline
\end{tabular}


At $40 \%$ strain, the values of stress at various strain rates are shown in table 1 . At $40 \%$ strain, a maximum stress of 1.3 $\mathrm{MPa}$ and 1.75 $\mathrm{MPa}$ have been observed in caprine muscle loaded along and perpendicular to the fiber direction respectively at a strain rate of $3700 \mathrm{~s}^{-1}$. Nie et al. [21] have reported a maximum stress of $0.3 \mathrm{MPa}$ and $0.35 \mathrm{MPa}$ at $30 \%$ strain for the porcine muscle loaded along and perpendicular to fiber direction at $2100 \mathrm{~s}^{-1}$. The similar trend of stress-strain curve in regard to the loading rate is observed in the literature on tensile testing of porcine muscle [24] and pig skin [28] at higher strain rates using polymeric SHPB. The caprine muscle response in the present study has been found to be stiffer than porcine muscle response reported by Nie et al. [30]. The tensile stress-strain response of caprine muscle tissue was found to be higher than that of porcine muscle tissue.

\section{CONCLUSIONS}

The uniaxial tensile stress-strain response of caprine (goat) muscle has been determined experimentally at varying strain rates ranging from 400 to $3700 \mathrm{~s}^{-1}$ using custom-made polymeric SHPB. The muscle tissues were reaped from lower furthest point of caprine and dainty annular examples were set up along and opposite to the fiber heading. The test pressure strain reactions are seen as non-straight and exceptionally subject to strain rate. The nonappearance of toe areas on ductile pressure strain bends demonstrates that muscle strands are straight and smooth toward the beginning of stacking. The greatest pliable burdens and strains increment altogether with increment in strain rates. It is likewise seen that at a similar strain rate, the example worry of caprine muscle along the opposite fiber (TR) course is higher than that along the fiber (AL) bearing. The utilization of polymeric bars in SHPB is reasonable for portrayal of muscle tissue at high strain rates.The setup developed can be used for testing soft tissues such as skin and muscle at high rates of loading. Experimental stress-strain data under tensile loading can be used in computational modeling of human body for impact based simulations of high rate scenarios.

\section{ACKNOWLEDGEMENTS}

The authors would like to acknowledge DBATU, Lonere for providing the research facility in the department of mechanical engineering. The authors are also thankful to M/S Shree Engineering, Baramati for providing technical support to develop customized SHPB setup and ARK Info-solutions Pvt. Ltd., Pune for their help during experiments.

\section{ABBREVIATIONS / NOMENCLATURE}

$\begin{array}{llll}\text { NI } & \text { National Instruments } & E & \text { Modulus of elasticity of pressure bars } \\ \text { SHPB } & \text { Split Hopkinson Pressure Bar } & L_{0} & \text { Length of pressure bar } \\ \text { PMHS } & \text { Post Mortem Human Subject } & \gamma(\omega) & \text { Propagation coefficient at frequency, } \omega \\ \varepsilon_{i} & \text { Incident pulse } & E^{*}(\omega) & \text { Complex Young's modulus of the bar } \\ \varepsilon_{r} & \text { Reflected pulse } & \tilde{\varepsilon}_{R_{-} n e w}(\omega) & \text { Reconstructed reflected strain at frequency, } \omega \\ \varepsilon_{t} & \text { Transmitted pulse } & \tilde{\varepsilon}_{T-n e w}(\omega) & \text { Reconstructed transmitted strain at frequency, }\end{array}$




$\begin{array}{llll}\varepsilon_{S}(t) & \text { Strain induced in specimen } & \varepsilon_{S}(t) & \text { Strain in the specimen at frequency, } \omega \\ \dot{\varepsilon}_{S}(t) \quad \text { Strain rate induced in specimen } & \dot{\varepsilon}_{S}(t) & \text { Strain rate in specimen at frequency, } \omega \\ \sigma_{S}(t) & \text { Stress induced in specimen } & \sigma_{S}(\omega) & \begin{array}{l}\text { Average stress in the specimen at frequency, } \\ \omega\end{array} \\ V_{s t} & \text { Impact velocity of striker bar } & \mathrm{c}(\omega) & \text { Phase velocity } \\ A_{0} & \text { Cross-sectional area of pressure bars } & \alpha(\omega) & \text { Attenuation coefficient } \\ C_{0} & \text { Sound velocity of polymer bars } & \mathrm{k}(\omega) & \text { Wave number } \\ A_{s} & \text { Initial cross-sectional area of specimen } & \mathrm{F}_{\text {elastic }} & \begin{array}{l}\text { Force due to elastic analysis by Bacon } \\ \text { approach }\end{array} \\ L_{s} & \text { Initial length of specimen } & & \begin{array}{l}\text { Force due to viscoelastic analysis by Bacon } \\ \text { approach }\end{array}\end{array}$

\section{REFERENCES}

1. L. Van, C. Lyons, C. Simms. (2008). Viscoelastic properties of passive skeletal muscle in compression: stress-relaxtaion behaviour and constitutive modeling, Journal of Biomechanics, 41, pp. 1555-1566.

2. H. Warhatkar, A. Chawla, S. Mukherjee, R. Malhotra. (2009). Experimental Study of Variation between Quasi-Static and Dynamic Load Deformation Properties of Medial Collateral Knee Ligaments, SAE Technical Papers, SAE World Congress, Detriot.

3. X. Zhai, W. Chen. (2018). Compressive Mechanical Response of Porcine Muscle at Intermediate $\left(10^{0} / \mathrm{s}-10^{2} / \mathrm{s}\right)$ Strain Rates, Experimental Mechanics, SEM.

4. X. Zhai, E. Nauman, Y. Nie, H. Liao et al. (2019). Mechanical response of human muscle at intermediate strain rates, Journal of Biomechanical Engineering. ASME. Pp. 1-18.

5. A. R. Kemper, A. C. Santago, J. D. Stitzel, J. L. Sparks and S. M. Duma. (2012). Biomechanical response of human spleen in tensile loading. Journal of Biomechanics, 45(2), pp. 348-355.

6. P. Gaur, A. Chawla, K. Verma, S. Mukherjee, S. Lalvani, R. Malhotra, and C. Mayer. (2016). Characterization of human diaphragm at high strain rate loading, Journal of the Mechanical Behavior of Biomedical Materials, 60, pp. 603-616.

7. Awate, Pankaj P., and Shivprakash B. Barve. "Formation and Characterization of Aluminium Metal Matrix Nanocomposites." Technology 64.557 (2015).International Journal of Mechanical and Production Engineering Research and Development (IJMPERD)9. 6, Dec 2019, 933-942

8. D. Mohan, J. W. Melvin. (1982). Failure properties of passive human aortic tissue. I-Uniaxial tension tests, Journal of Biomechanics, 15(11), pp. 887-902.

9. J. Hopkinson. (1901). Further experiments on the rupture of iron wire, Article 39, B. Hopkinson (ed.), Original Papers-by the late John Hopkinson, Vol. II, Scientific Papers, Cambridge. 
10. B. Hopkinson. (1914). A Method of Measuring the Pressure Produced in the Detonation of High Explosives or by the Impact of Bullets, Philosophical Transactions of the Royal Society of London: Series A, Vol. 213, pp. 437-456.

11. R. Davis. (1948). A critical study of the Hopkinosn pressure bar, Philosophical Transactions of the Royal Society of London: Series A, 240, pp. 375-457.

12. H. Kolsky. (1949). An investigation of the mechanical properties of materials at very high rates of loading, Proceedings of the Physical Society-Section B 62, pp. 676-700.

13. E. Davies, S. Hunter. (1963). The Dynamic Compression Testing of Solids by the Method of the Split Hopkinson Pressure Bar, Journal of the Mechanics and Physics of Solids, 11(3), pp. 155-179.

14. F. Pervin, W. Chen, T. Weerasooriya. (2010). Dynamic compressive response of renal cortex, International Journal of Structural Changes in Solids, 2(1), pp. 1-7.

15. F. Pervin, W. Chen, T. Weerasooriya. (2011). Dynamic compressive response of bovine liver tissue, Journal of Mechanical Behavour Biomedical Materials, 4, pp. 76-84.

16. Patel, Mehul D., et al. "Adaptive physiological and biochemical responses of dairy animals to heat stress: a review." International Journal of Applied and Natural Sciences 5.1 (2016): 107-116.

17. F. Pervin, W. Chen. (2009). Dynamic mechanical response of bovine gray and white matter brain tissues under compression, Journal of Biomechanics, 42, pp. 731-735.

18. J. Chen, S. Patnaik, R. Prabh, L. Proddy et al. (2019). Mechanical response of porcine liver tissue under high strain rate compression, Biengineering, 6( 49), pp. 1-16.

19. K. Comley, N. Fleck. (2012). The compressive response of porcine adipose tissue from low to high strain rate, International Journal of Impact Engineering, 46, 1-10.

20. Irfan, Muhammad, et al. "Ethnomedicinal and traditional knowledge of phanerogames of Tehsil Munda, district lower Dir, Khyber Pakhtunkhwa, Pakistan." International Journal of Biosciences 13.4 (2018): 208-218.

21. B. Butler, C. Bo, A. Tucker, A. Jardine et al.(2014). Mechanical and histological characterization of trachea tissue subjected to blast-type pressures, IOP Journal of Physics: Conference Series, $18^{\text {th }}$ APS-SCCM and 24th AIRAPT, 500.

22. B. Sanborn, X. Nie, W. Chen, T. Weerasooriya. (2013). High strain-rate pure shear and uniaxial compressive response of porcine lung tissue, Journal of Applied Mechanics, 80, pp. 11-29.

23. B. Song, W. Chen, Y. Ge, T. Weerasooriya. (2007). Dynamic and quasi-static compressive response of porcine muscle, Journal of Biomechanics, 40, 2999-3005.

24. S. C. Van, D. S. Cronin, G. Brodland. (2006). High strain rate compressive properties of bovine muscle tissue determined using split Hopkinson bar apparatus, Journal of Biomechanics, 39, pp. 1852-1858.

25. B. Karthikeyan, S. Mukherjee, A. Chawala, R. Malhotra. (2012). Dynamic compressive response of passive human muscles using Split Hopkinson Pressure Bar, Indian Journal of Biomechanics, 1(3), pp. 20-28.

26. Gawdaman, G., Robinson JJ Abraham, and K. S. Gnanalakshmi. "Analysis of metallic residues in goat tissues through ICPOES." International Journal of Agricultural Science and Research (IJASR) 5.3 (2015): 279-285.

27. S. H. Kadhane, H. N. Warhatkar. (2019). Dynamic Stress-Strain Compressive Response of Soft Tissue using Polymeric Split Hopkinson Pressure Bar, International Journal of Innovative Technology and Exploring Engineering, 8(9), pp. 341-347. 
28. X. Nie, J. Cheng, W. Chen, T. Weerasooriya. (2011). Dynamic tensile response of porcine muscle, Journal of Applied Mechanics, 78(2), pp. 021009-1-021009-5.

29. B. Z. Wang, Y. X. Zhen, S. S. Hu. (2010). Dynamic tensile properties of procine ham muscle, Explosion and Shock Waves, 30 , pp. 33-38.

30. M. Cheng, W. Chen, T. Weerasooriya. (2009). Mechanical behavior of bovine tendon with stress and loading rate effects, Advanced Theoretical Applied Biomechanics, 2(2), pp. 59-74.

31. O. Shergold, N. Fleck, D. Radford. (2006). The uniaxial stress versus strain response of pig skin and silicone rubber at low and high strain rates, International Journal of Impact Engineering, 32, pp. 1384-1402.

32. J. Lim, J. Hong, W. Chen, T. Weerasooriya. (2011). Mechanical response of pig skin under dynamic tensile testing, International Journal of Impact Engineering, 38, pp. 130-135.

33. V. P. W. Shim, J. F. Liu, V. S. Lee. (2006). A technique for dynamic tensile testing of ligaments from the human cervical spine ligaments, Experimental Mechanics, 46, pp. 77-89.

34. M. Trexler, A. Lennon, A. Wickwire et al. (2011). Verification and implementation of a modified split Hopkinson pressure bar technique for characterizing biological tissue and soft biosimulant materials under dynamic shear loading, Journal of Mechanical Behavior of Biomedical Materials, 4, 1920-928.

35. W. Chen. (2016). Experimental methods for characterizing dynamic response of soft materials, Journal of Dynamic Behavior of Materials, 2, pp. 2-14.

36. C. Bacon. (1998). An experimental method for considering dispersion and attenuation in a viscoelastic Hopkinson bar, Experimental Mechanics, 38, pp. 242-249.

37. S. H. Kadhane, H. N. Warhatkar. (2017). Design and development of an integrated compressive-tensile polymeric split Hopkinson pressure bar setup, Procedia Engineering, 173, pp. 702-709.

38. G. Mutungi, P. Purslow, C. Warkup. (1995). Structural and Mechanical Changes in Raw and Cooked Single Porcine Muscle Fibers Extended to Fracture, Meat Science,40, pp. 217-234.

39. T. M. Best, J. MacElhaney, E. G. William, B. S. Mayers. (1994). Characterization of paasive response of live skeletal muscle using quasi-linear theory of viscoelasticity, Journal of Biomechanics, 27, 413-419.

40. G. A. Meyer, A. D. McCulloch, R. L. Lieber. (2011). A nonlinear model of passive muscle viscosity, Journal of Biomechanical Engineeirng, 133, 091007.

41. K. Wang, R. McCarter, J. Wright, J. Beverly, R. Ramirez-Mitchell. (1993). Viscoelasticity of the sarcomere matrix of skeletal muscles: The titin-myosin composite filament is a dual-stage molecular spring,Biophys Journal, 64, 1161-1177.

42. M. L. Bartoo, W. A. Linke, G. H. Pollack. (1997). Basis of passive tension and stiffness in isolated rabbit myofibrils, Americal Journal of Physiology, 273, C266-C276. 